\title{
L'écologie, fondement et support de la lutte biologique
}

\author{
Introduction générale
}

\author{
par E. BILIOTTI \\ Département de Zoologie de I'I.N.R.A., C.N.R.A., F. 78 -Versailles
}

\begin{abstract}
Résumé
La nécessité de tenir compte de l'écologie des populations avant d'entreprendre des opérations de lutte biologique est illustrée par l'irrégularité des résultats obtenus dans les premières expériences d'introduction d'insectes entomophages.

Des progrès remarquables dans la lutte biologique ont été réalisés au cours des 20 dernières années. Ils ont été rendus possibles par:

- la généralisation et l'amélioration des études sur les complexes biocénotiques,

- le développement des recherches sur les mécanismes des interactions interspécifiques et intraspécifiques,

- la mise au point de méthodes d'appréciation quantitative du niveau de population des différentes espèces,

- les essais d'interprétation mathématique des fluctuations de population.

La lutte biologique classique contre les insectes vecteurs est en retard par rapport à ce qui a été réalisé dans le secteur agricole et forestier, par contre les études sur les méthodes génétiques de lutte ont été particulièrement développées dans ce domaine.
\end{abstract}

\section{Summary}

Ecology, basic of biological control.

The lack of knowledge on population ecology explains most of the failures in various attempts of biological control. 
Very fine progresses have been obtained during the past 20 years in the field of :

- study of biocenotic communities,

- research on the mecanisms of inter and intra specific competition,

- quantitative evaluation of the levels of populations,

- mathematical analysis of population changes.

Examples of success in biological control of insect vectors are scare but basic studies on the possibility of genetic control have been promisingly developed.

Bien que le sujet général de ce colloque soit la lutte biologique, l'un des aspects principaux de cette méthode ne figure pas à l'ordre du jour ; en effet, l'utilisation des animaux entomophages (insectes ou autres) ne sera pas envisagée. Cependant, ces organismes ont été les premiers utilisés en lutte biologique, et, s'il n'a pas paru utile de discuter de leur rôle dans le cadre de ce colloque, ils peuvent, du moins, nous servir à illustrer le premier thème de nos débats sur l'importance des études écologiques.

Les premiers succès de la lutte biologique ont été obtenus grâce à l'introduction d'insectes entomophages dans un milieu où ils n'existaient pas, et pour lutter contre des ravageurs eux-mêmes introduits, et ayant de ce fait, échappé aux ennemis naturels de leur pays d'origine.

La conception de base de la méthode était simple, et ne nécessitait pas, a priori, une connaissance approfondie de l'écosystème. Le succès paraîssait dépendre essentiellement des «qualités » intrinsèques du parasite ou du prédateur choisi : fécondité, longévité, voltinisme, activité prédatrice, etc., et les auteurs se sont attachés à établir un «portrait-robot» de l'entomophage idéal à employer en lutte biologique. Les discussions ont porté plus sur les mérites théoriques respectifs des différentes catégories d'entomophages, parasites ou prédateurs, que sur les contraintes imposées par le milieu à une espèce déterminée (Thompson, 1928).

Cependant, les succès spectaculaires du départ ont été suivis d'un certain nombre de déceptions, et on a rapidement constaté que des espèces, apparemment dotées de toutes les qualités requises, se montraient incapables d'abaisser, de façon permanente, le niveau de population de leurs hôtes au-dessous du seuil recherché. L'explication de ces échecs a montré que l'on n'avait pas suffisamment tenu compte de la nécessité pour l'organisme utile, de s'adapter à un milieu complexe, où il était soumis, non seulement à l'action des facteurs climatiques, mais à de multiples interactions avec les autres êtres vivants présents dans l'écosystème. On a notamment compris que les facteurs climatiques n'agissaient pas seulement en «modelant» le cycle annuel d'une espèce considérée, mais aussi en modifiant, de façon parfois extrêmement importante, le déroulement des phénomènes de compétition entre les espèces. Il ne suffisait donc pas de choisir une espèce utile en fonction du diagramme climatique de son pays d'ori- 
gine et de celui de la région dans laquelle on voulait l'introduire, mais il convenait d'étudier beaucoup plus finement le rôle des facteurs physico-chimiques du milieu, dans chaque situation particulière. Par ailleurs, on avait tendance à considérer au départ, que chaque espèce d'insecte constituait une entité stable ; on s'est aperçu progressivement, que les populations distinctes d'une même espèce possédaient des potentialités différentes suivant les régions. Ceci est valable, non seulement pour des zones géographiques disjointes, mais également à l'échelle de biotopes voisins et de taille parfois réduite.

C'est donc en termes d'écologie des populations, qu'il convient de raisonner, et l'étude approfondie de la génétique des populations naturelles s'impose. Ces considérations sont évidemment valables pour toutes les méthodes de lutte, puisqu'il est nécessaire dans tous les cas, de comprendre les phénomènes qui régissent les fluctuations du niveau de population des espèces nuisibles ou gênantes, si l'on veut être en mesure de s'opposer à leur pullulation.

C'est cependant dans le domaine de la lutte biologique que les progrès les plus remarquables ont été réalisés au cours des vingt dernières années ; on peut les résumer de la façon suivante :

— généralisation et amélioration des études sur les complexes biocénotiques,

- recherches sur les mécanismes des interactions interspécifiques et intraspécifiques,

- mise au point de méthodes d'appréciation quantitative du niveau de population des différentes espèces,

- essai d'interprétation mathématique des fluctuations de population.

Dans ce dernier domaine, les premiers travaux consistaient essentiellement en l'établissement de modèles théoriques construits à partir d'un certain nombre de postulats portant sur les propriétés biologiques et éthologiques des organismes en présence. Cela a conduit à des théories parfois très séduisantes ; mais lorsqu'on a voulu les vérifier sur quelques cas précis, on a eu immédiatement des déboires, qui tenaient au fait que les postulats de départ étaient généralement faux. La tendance qui se développe actuellement consiste, au contraire, à appliquer toutes les ressources de l'analyse mathématique aux relevés numériques obtenus dans l'étude approfondie et permanente, pendant plusieurs générations, de populations naturelles régulièrement échantillonnées (Griffiths et Holling, 1969). Je pense que c'est à partir des «tables de vie » que l'on pourra essayer valablement d'interpréter les fluctuations de populations d'insectes pour être en mesure de les prévoir, et d'en modifier le déroulement à notre profit. A l'heure actuelle, les progrès les plus marquants ont été faits dans le domaine de l'entomologie forestière (Northeastern Forest Experiment Station, 1969), mais nous disposons d'assez peu d'éléments pour ce qui concerne les insectes vecteurs.

Dans ce domaine particulier, l'inventaire biocénotique des parasites et des prédateurs est poursuivi depuis de longues années, et a fait l'objet d'un certain nombre de revues générales comme celle de Jenkins (1964). 
Parmi les insectes entomophages s'attaquant aux vecteurs, on ne trouve pas d'espèces parasites et aucun représentant des familles les mieux connues et les mieux utilisées en entomologie agricole. Par contre, les prédateurs sont extrêmement abondants (198 prédateurs de larves sur 448 recensés par Jenkins, et 63 prédateurs d'adultes sur 100 répertoriés), et ils appartiennent à des espèces très largement polyphages. Le rôle de telles espèces est encore mal connu, et leur utilisation dans le domaine agricole n'a encore fait l'objet que d'un nombre restreint d'essais. Nous ne sommes pas toujours capables, dans toute la série d'espèces soumises à l'action d'un prédateur déterminé, de reconnaître, à un moment donné, celle dont le développement sera effectivement freiné par son action.

Dans le cas de la plupart des insectes vecteurs, nous n'avons pas dépassé la phase de l'inventaire des facteurs potentiels de régulation, et seule une étude écologique approfondie pourra nous permettre de détecter, puis de mettre en œuvre, les organismes vivants capables d'exercer une limitation permanente du niveau de population des espèces à combattre.

Beaucoup de tentatives d'utilisation d'arthropodes utiles ont eu lieu sans qu'on se soit préoccupé d'étudier à fond les mécanismes en cause, ce qui nous interdit d'en tirer, à l'heure actuelle, des enseignements valables. Ceci est particulièrement regrettable lorsqu'il s'agit de formes présentant des potentialités très intéressantes comme les Megarhinus (Newkirk, 1947).

Ces moustiques dont les larves sont prédatrices de celles des autres espèces, ont le très grand avantage d'occuper à peu près la même «niche \& écologique que certains des vecteurs les plus nocifs dans la région du Pacifique. Ils ont fait l'objet de plusieurs introductions, notamment aux Hawaï (Paine, 1934, Bonnet et Hu, 1952, Peterson, 1956) ; les techniques de manipulation ont été mises au point, certaines acclimatations ont été constatées, mais l'insuffisance de l'analyse écologique ne permet pas, à l'heure actuelle, de tirer un plein profit des efforts réalisés. Il serait cependant possible d'améliorer un certain nombre de résultats obtenus dans le passé. Des centaines de travaux répertoriés par Gerberich (1946) et par Gerberich et Laird (1968) portent sur l'utilisation des poissons prédateurs, et parmi ceux-ci, on a eu très fréquemment recours à Gambusia holbrooki qui joue actuellement un rôle important dans toutes les eaux permanentes du midi de la France. On pourrait très certainement améliorer les techniques de distribution, pour coloniser les eaux temporaires, et il serait possible de tirer parti de la différenciation écologique subie par l'espèce depuis sa première introduction.

En effet, cette espèce, originaire du Sud des Etats-Unis, a été importée en Espagne en 1921, puis en Corse en 1924, et elle a fait l'objet d'un élevage permanent au Centre de Recherches agronomiques de Provence d'Antibes depuis 1933. Très largement distribuée dans tout le Sud de la France, elle a très probablement développé, en quelques points, des écotypes particuliers capables de résister au froid (Maillet, 1954) qui pourraient servir de base à de nouvelles multiplications, en vue de l'extension de sa zone d'activité. 
En outre, certains prédateurs présentent des potentialités d'adaptation au milieu dont on n'a pas encore tiré tout le parti possible. C'est ainsi que nos collègues de Californie (Bay, 1965, 1966) ont développé, ces dernières années, des travaux im sortants sur l'utilisation d'espèces dont les œufs sont capables de supporter une période de dessèchement, et de reprendre leur développement dès le retour de l'eau.

Le domaine des maladies des vecteurs fera l'objet d'un développement particulier. Nous y trouverons encore une insuffisance des données écologiques de base, et un retard par rapport au secteur agricole (Debach, 1964) ou forestier.

Par contre, chez les insectes vecteurs, la mise en évidence des différentes potentialités présentées par les populations naturelles, a été particulièrement poussée, et il a été rapidement possible d'envisager d'utiliser ces différences dans le domaine de la «lutte génétique». Notre colloque accordera donc une place importante aux bases génétiques, et à la mise au point pratique des méthodes développées dans ce secteur. Nous espérons ainsi créer l'occasion d'un large échange de vue, et jeter les bases de coopérations fructueuses pour promouvoir la lutte biologique contre les vecteurs sous toutes les formes possibles.

\section{Bibliographie}

BAY (E. C.), 1965. - Instant fish! A new tool for mosquito control ? Pest Control Mag., apr.

-, 1966. - Adaptation studies with the argentine pearl fish, Cynolebias bellottii, for its introduction into California. Copeia, 4: 839-846.

BonNeT (D. D.), Hu (S. M. K.), 1952. - The introduction of Toxorhynchites brevipalpus Theobald into the territory of Hawaii. Proc. Haw. Entomol. Soc., 14, 237-242.

De Bach (P.), Schlinger (E. I.), edit,, 1964. - Biological control of insect pests and weeds. Chapman \& Hall, Londres, 844 p.

GERBERICH (J. B.), 1946. - An annotated bibliography of papers relating to the control of mosquitoes by the use of fish. Amer. Midl. Natural., 36, 87-131.

-, LAIRD (M.), 1968. - Bibliography of papers relating to the control of mosquitoes by the use of fish. An annotated bibliography for the years 1901-1966. F.A.O. Fish. tech. Pap., 75, 70 p.

Griffiths (K. G.), Holling (C.S.), 1969. - A competition submodel for parasites and predators. Can. Entomol., 101 : 785-818.

JENKINS (D. W.), 1964. - Pathogens, parasites and predators of medically important arthropods. Annotated list and bibliography. Bull. Org. Mond. Santé, 30, suppl., 150 p.

Maillet (P.), 1954. - Sur un élevage du Gambusia holbrooki à la Station biologique des Eyzies. Vie et Milieu, 5 : 459-464.

NewKIRK (M. R.), 1947. - Observations on Megharinus splendens Wiedemann with reference to its value in biological control of other mosquitoes (Diptera, Culicidae). Ann. Entomol. Soc. Amer., 40 : 522-527. 
NORTHEASTERN FOREST EXPERIMENT STATION, 1969. - Forest insect population dynamics workshop. N.E. Forest Exp. Sta. Upper Darby, PA, 126 p. (U.S.D.A. Forest Serv. Res. Paper, NE 125).

PaINE (R. W.), 1934. - The introduction of Megharinus mosquitos in Fiji. Bull. Entomol. Res., $25: 1-31$.

Peterson (G. D.), 1956. - The introduction of mosquitoes of the genus Toxorhynchites into american Samoa. J. econ. Entomol., 49: 786-789.

Thompson (W. R.), 1928. - On the relative value of parasites and predators in the biological control of insect pests. Bull. Entomol. Res., 19: 343-350. 